\title{
Chylothorax Associated with Congenital Complete Atrioventricular Block
}

\author{
Shuhei Fujino, $\mathrm{MD}^{1}$ Hidehiko Maruyama, MD, $\mathrm{PhD}^{1}$ \\ Tetsuya Isayama, MD, $\mathrm{PhD}^{1}$ Yushi Ito, $\mathrm{MD}^{1}$ \\ ${ }^{1}$ Division of Neonatology, Center for Maternal-Fetal, Neonatal and \\ Reproductive Medicine, National Center for Child Health and \\ Development, Tokyo, Japan \\ 2 Division of Cardiology, National Center for Child Health and \\ Development, Tokyo, Japan
}

Keiko Tsukamoto, MD ${ }^{1}$ Hiroshi Ono, MD $^{2}$

\begin{abstract}
Address for correspondence Yushi Ito, MD, Division of Neonatology, Center for maternal-Fetal, Neonatal and Reproductive Medicine, National Center for Child Health and Development, 2-10-1, Okura, Setagaya-ku, 157-0074, Tokyo, Japan (e-mail: itoh-y@ncchd.go.jp).
\end{abstract}

Am J Perinatol Rep 2020;10:e403-e407.

\begin{abstract}
\section{Keywords}

- congenital complete atrioventricular block

- congenital chylothorax

- anti-SS-A/Ro antibody

- prednisolone

- octreotide

- coagulation factor XIII

Introduction Congenital complete atrioventricular block (CCAVB) associated with congenital chylothorax is a rare finding that has been reported in only one case in the literature. We report here the case of an infant with CCAVB complicated by congenital chylothorax.

Patient Report We present the case of a male neonate with a birth weight of $2114 \mathrm{~g}$. Fetal bradycardia and right pleural effusion were detected at gestational age of 22 weeks. Maternal serum levels of anti-Sjögren's-syndrome-related antigen A autoantibody were high $(4840 \mathrm{U} / \mathrm{mL})$. The neonate was delivered at gestational age of 33 weeks; a temporary external pacemaker was placed immediately after birth that resulted in an improved cardiac output. Milk-colored pleural effusion increased in volume together with the initiation of breast milk feeding. Lymphocytosis and high triglyceride levels in the pleural fluid led to the diagnosis of chylothorax. The pleural effusion resolved in response to prednisolone, octreotide, and total parenteral nutrition.

Discussion The causal relationship between CCAVB and congenital chylothorax can be explained by considering the damage to the lymphatic vessels secondary to inflammation due to maternal autoantibodies and venous congestion due to bradycardia.

Conclusion In any case of CCAVB associated with atypical pleural effusion, one must consider the possibility of congenital chylothorax.
\end{abstract}

Fetal congenital complete atrioventricular block (CCAVB) has been reported in 1 to $2 \%$ of infants with positive maternal anti-Sjögren's-syndrome-related antigen $\mathrm{A}$ (anti-SS-A/Ro) antibody and is a result of damage to the cardiac impulse conducting system. ${ }^{1}$ Congenital chylothorax results from the accumulation of lymphatic fluid in the thoracic cavity; this disorder is reported in $1 / 10,000$ to 15,000 live births. ${ }^{2}$ CCAVB can be complicated by tran-

received

March 8, 2020

accepted

May 29, 2020
DOI https://doi.org/

10.1055/s-0040-1715178. ISSN 2157-6998. sudative pleural effusion or hydrops fetalis resulting from cardiac failure secondary to bradycardia. CCAVB diagnosed in association with chylothorax is an extremely rare event and has been reported in only one case with trisomy 21 previously. ${ }^{3}$ However, the detailed clinical course, treatment, and underlying pathogenic mechanism remain unclear. We report here a case of fetal and neonatal CCAVB complicated by chylothorax.
Copyright $\odot 2020$ by Thieme Medical Publishers, Inc., 333 Seventh Avenue, New York, NY 10001, USA. Tel: +1(212) 760-0888.
License terms

()(1) $\Theta \circledast$ 


\section{Patient Presentation}

The patient was a male neonate born at the gestational age of 33 weeks and 3 days with birth weight of $2114 \mathrm{~g}$.

\section{Pregnancy and Delivery Course}

At gestational age of 22 weeks, bradycardia was diagnosed, with independent beating of the atria $(86 / \mathrm{min})$ and ventricles (47/min; - Fig. 1A). The fetus was diagnosed with CCAVB. Right pleural effusion was detected concurrently by fetal ultrasonography (-Fig. 1B). Maternal serum anti-SS-A/Ro antibody was positive at $4840 \mathrm{U} / \mathrm{mL}$ (reference value $<10 \mathrm{U} / \mathrm{mL}$ ). ${ }^{4}$ The mother reported no history of systemic lupus erythematosus (SLE) or Sjögren's syndrome. Ritodrine hydrochloride and betamethasone were administered to the mother starting at fetal gestational age of 22 weeks; the pleural effusion diminished gradually and temporarily disappeared (gestation age 27-32 weeks), although the fetal heart rate did not improve. At gestational age of 32 weeks, the ventricular rate decreased to $37 / \mathrm{min}$ in association with the re-emergence of right pleural effusion. The fetus was diagnosed with hydrops fetalis and was delivered by cesarean section at gestational age of 33 weeks to provide appropriate treatment for CCAVB and the pleural effusion.

\section{Postnatal Course}

At birth, physical signs included marked subcutaneous edema. A chest radiograph revealed right pleural effusion. Tracheal intubation was performed in response to acute respiratory distress and the right pleural effusion was drained. Cardiac ultrasonography and electrocardiographic findings revealed a ventricular rate of $37 / \mathrm{min}$. Echocardiography revealed a left ventricular ejection fraction (LVEF) of $56 \%$ and a cardiac index (CI) of $2.0 \mathrm{~L} / \mathrm{m}^{2} / \mathrm{min}$ (reference value: $4.0-5.0 \mathrm{~L} / \mathrm{m}^{2} / \mathrm{min}$ ). ${ }^{5} \mathrm{High}$ levels of serum anti-SS-A/Ro antibodies were detected 1,110 $\mathrm{U} / \mathrm{mL}$ (reference value $<10$ ). ${ }^{4}$ Chromosomal evaluation indicated a normal karyotype (46, XY). A temporary external pacemaker was surgically placed on ventricular external wall immediately after birth (-Fig. 1C). Temporary external pacing resulted in increased LVEF to $70 \%$ and $\mathrm{CI}$ to 3.0 to 4.0 $\mathrm{L} / \mathrm{m}^{2} /$ min during the first week of life. Although the temporary pacemaker improved heart rate and cardiac output, milkycolored pleural effusion accumulated at an increasing rate up to $300 \mathrm{~mL} /$ day, correlating with the initiation of breast milk feeding at 3 days age (-Fig. 1D). The pleural effusion was notable for a high lymphocyte count (lymphocyte of $3,350 / \mu \mathrm{L}$, representing $\sim 97 \%$ of the total white cell count of $3625 / \mu \mathrm{L}$ ), a relatively high triglyceride level $(60 \mathrm{mg} / \mathrm{dL}$; serum level at $51 \mathrm{mg} / \mathrm{dL}$ ), relatively high total immunoglobulin G (IgG) ( $239 \mathrm{mg} / \mathrm{dL}$; serum level at $298 \mathrm{mg} / \mathrm{dL}$; pleural fluid/serum $(\mathrm{P} / \mathrm{S})$ ratio of 0.80$)$, high total protein $(2.8 \mathrm{~g} / \mathrm{dL})$, and a high level of lactate dehydrogenase (LDH) (856 IU/L). Based on these findings, the pleural effusion was diagnosed as chylothorax. Breast milk feeding was discontinued and switched to medium chain triglyceride-containing formula; this had no impact on the effusion. The pleural effusion disappeared within 3 weeks in response to total parenteral nutrition, prednisolone (maximum of $2.0 \mathrm{mg} / \mathrm{kg} / \mathrm{day}$ ), octreotide (maximum of $6.0 \mu \mathrm{g} / \mathrm{kg} / \mathrm{h}$ ), and coagulation factor XIII (30 units/kg/day for 5 days); this also facilitated critical weight gain. A permanent pacemaker was placed at 41 days when the neonate reached a weight of $2520 \mathrm{~g}$. Pleural effusion was no longer detected after placement of a permanent pacemaker. A summary of clinical course is presented in $\mathbf{- F i g} \mathbf{2}$.
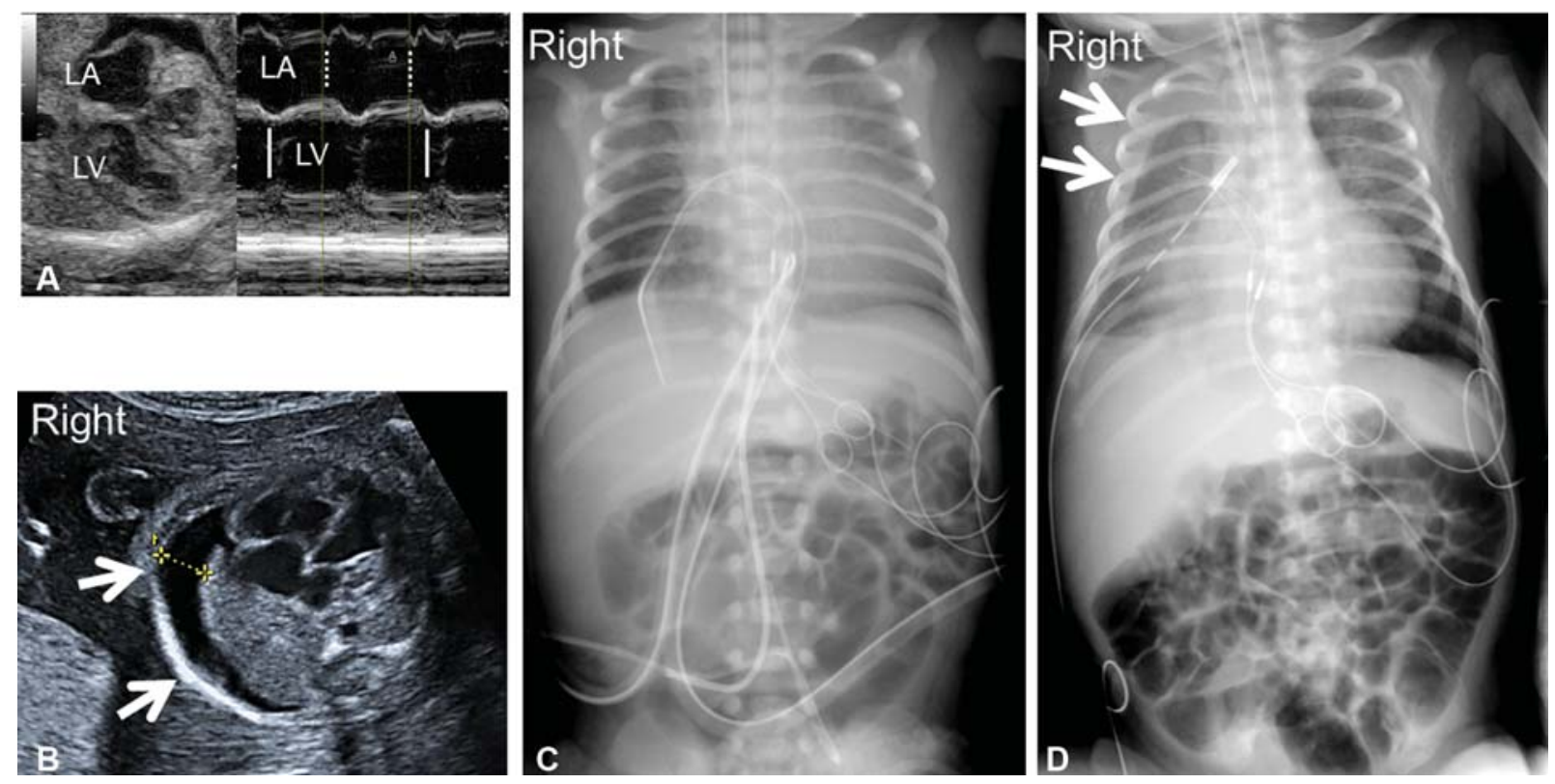

Fig. 1 Imaging findings from fetal to the neonatal period. (A) Fetal M-mode ultrasound imaging at gestational age of 22 weeks. The dotted line indicates the left atrium at end diastole, and the solid line indicates the left ventricle at end diastole. The atrial heart rate was $86 /$ minute, and the ventricular heart rate was 47/minute. (B) Fetal B-mode ultrasound imaging at 22 weeks of gestation. Right pleural effusion is as indicated by the white arrow. (C, D) Chest radiograph findings after birth. (C) The right thoracic drainage and the temporary pacemaker leads were placed at birth (age 0 days). (D) Right pleural effusion subsequently increased upon initiation of breast milk feeding at age 7 days (white arrow). 


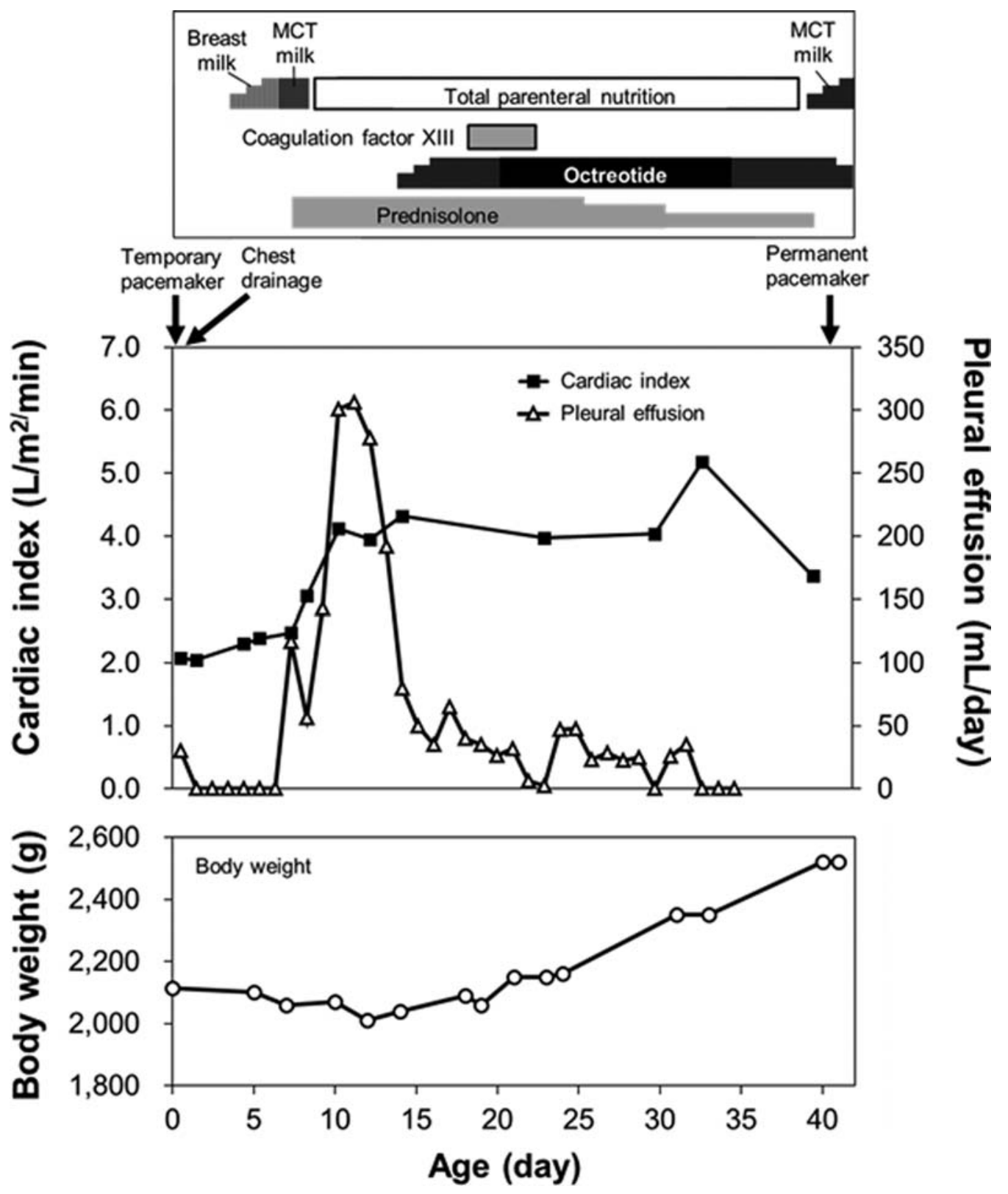

Fig. 2 Timeline of the clinical course from birth to permanent pacemaker placement (age 41 days). Upper panel: feeding, treatments, and contents of the pleural effusion. Middle panel: cardiac index $\left(\mathrm{L} / \mathrm{m}^{2} / \mathrm{min}\right)$ and pleural effluent volume ( $\mathrm{mL} /$ day). Lower panel: body weight $(\mathrm{g})$. MCT milk, milk containing medium chain triglycerides.

\section{Discussion}

In this case, the pleural effusion was not transudative as one might expect secondary to bradycardia and cardiac failure but chylous in nature. Chylothorax was diagnosed according to the following four characteristic findings, each discussed in turn.

- Prominent lymphocytosis and relatively high triglyceride levels were detected in the pleural effusion. ${ }^{2}$ Among neonates, chylothorax can be distinguished from pleural transudate and pleural exudate by the leukocyte count in pleural effusion is $>1,000 / \mu \mathrm{L}^{2}$ with a lymphocyte fraction above $80 \%,{ }^{2}$ and elevated triglyceride levels in the pleural effusion above 100 to $110 \mathrm{mg} / \mathrm{dL}$ or above levels detected in serum; however, triglycerides are not present unless enteral feeds are initiated. ${ }^{2}$ It is also informative if the pleural effusion is milk-colored, with total protein levels higher than $2.5 \mathrm{~g} / \mathrm{dL},{ }^{6}$ a $\mathrm{P} / \mathrm{S}$ ratio of $\operatorname{IgG}>0.60{ }^{7}$ and $\mathrm{LDH}$ levels higher than $110 \mathrm{IU} / \mathrm{L}{ }^{2,6}$

- Pleural effusion accumulation was consistently unilateral (right side). Chylothorax is detected most often on the right side of the thorax; this is probably related to the anatomy of the thoracic duct. ${ }^{8}$ By contrast, transudative pleural effusion as a sign of fetal heart failure or hydrops fetalis typically develops bilaterally.

- Pleural effusion was exacerbated after the initiation of breast milk feeding despite improvement in heart rate and cardiac output. In cases of transudative pleural effusion, pleural effusion typically responds to an improvement in 
cardiac function. By contrast, chylothorax often increases after initiating enteral feeding. ${ }^{2}$

- A temporary improvement in fetal pleural effusion was detected after betamethasone administration to the mother, despite no improvements in fetal heart rate. Saito et $\mathrm{al}^{3}$ reported that maternal treatment with betamethasone was an effective at reducing pleural effusion in congenital chylothorax.

The relationship between CCAVB and chylothorax has not been clarified. Chylothorax can be the result of a surgical procedure, such as open-heart surgery, that results in injury to the thoracic duct, ${ }^{2}$ but it is unlikely to be caused by temporary pacemaker placement in a procedure limited to the anterior thorax. Although it is possible that these two rare diseases occurred together by coincidence, a causal relationship may be considered:

1. Chylothorax may result from damage to the lymphatic vessels secondary to venous congestion due to bradycardia and heart failure. ${ }^{9}$ Approximately $30 \%$ of cases of congenital chylothorax are accompanied by fatal complications such as heart failure. In these cases, chylothorax results from venous stasis and pressure injury sustained by the lymphatic system. ${ }^{9}$ Although the lymph vessels damaged by venous congestion are not repaired by pacemaker placement, we hypothesize that the resolution of heart failure due to bradycardia and elimination of venous stasis may prevent further deterioration and will permit the pleural effusion to resolve.

2. Lymphatic vessel inflammation may be caused by maternal anti-SS-A/Ro autoantibodies. There are several reports documenting the clinical course of adult cases with SLE who developed chylous pleural and/or peritoneal effusions in association with high serum levels of autoantibody including anti-SS-A/Ro autoantibodies. ${ }^{10-12}$ In these reports, the authors speculated that autoantibody interactions with the lymphatic system may result in inflammation, increased lymphatic vasopermeability associated with inflammation, and elevated internal pressure due to collapse of the lymphatic vessels. In some of these previous cases, treatment with corticosteroids or other immunosuppressant agents resulted in at least partial resolution of the chylous effusion. ${ }^{10-12}$ Although the mother in this case report was not diagnosed with SLE, high levels of the serum anti-SS-A/Ro autoantibody were detected in both the mother and her neonatal child.

Although we cannot identify a definitive, causal relationship, standard treatment for chylothorax was effective and led to weight gain and permitted placement of a permanent pacemaker at 41 days. Malnutrition and immunological weakness resulting from protein/immunoglobulin loss from pleural effusion, intolerance of enteral feeding, and preterm low birth weight are the potential risk factors for infection that may interfere with permanent pacemaker placement. Therefore, we used temporary ex- ternal pacemaker at birth and attempted to reduce chylous effusion first. Appropriate treatment for chylothorax such as total parenteral nutrition, ${ }^{2}$ octreotide, ${ }^{2}$ prednisolone, ${ }^{13}$ and coagulation factor XIII ${ }^{14}$ was effective with a subsequent satisfactory course. Total parenteral nutrition and octreotide may serve to decrease lymphatic flow. ${ }^{2}$ Coagulation factor XIII may be involved in promoting tissue regeneration, repair of damaged lymphatic vessels, and may have proliferative and antiapoptotic effects on vascular endothelial cells. ${ }^{14}$ Likewise, corticosteroids may be effective for chylothorax because of their lymphatic vasoconstrictive effects and the reduction in lymphatic flow due to increasing plasma osmotic pressure. ${ }^{13}$ Moreover, if the maternal autoantibodies have a role in promoting the pathology that results in chylothorax, the anti-inflammatory effects of corticosteroid may also serve to decrease pleural effusion.

\section{Conclusion}

The causal relationship between CCAVB and chylothorax in this case may relate to damage of the lymphatic vessels due to bradycardia and venous congestion as well as maternal autoantibodies. In any case of CCAVB associated with an atypical pleural effusion, it will be important to consider the complication of congenital chylothorax.

Conflict of Interest

The authors declare no competing interests.

\section{Acknowledgments}

The authors would like to thank Enago (www.enago.jp) for the English language review. No financial support or personal assistance was received for this work.

\section{References}

1 Michaëlsson M, Engle MA. Congenital complete heart block: an international study of the natural history. Cardiovasc Clin 1972;4 (03):85-101

2 Keller BA, Hirose S, Farmer DL. Surgical Disorders of the Chest and Airways. In: Gleason CA, Juul SE. eds. Avery's Diseases of the Newborn. 10th edition. Philadelphia: Elsevier Saunders; 2018: 695-723

3 Saito K, Tao K, Mizukaki N, et al. A case of Down syndrome baby, with fetal hydrops and congenital complete AV block. . [Article in Japanese]J Jpn Soc Perin Neon Med 2013;49:1073-1077

4 Tanaka N, Muro Y, Sugiura K, Tomita Y. Anti-SS-A/Ro antibody determination by indirect immunofluorescence and comparison of different methods of anti-nuclear antibody screening: evaluation of the utility of HEp-2 cells transfected with the 60 . kDa SS-A/Ro as a substrate. Mod Rheumatol 2008;18(06): 585-592

5 Boville B, Young LC. Quick Guide to Pediatric Cardiopulmonary Care. Irvine: Edwards Lifesciences; 2015:3-78

6 Lopez-Gutierrez JC, Tovar JA. Chylothorax and chylous ascites: management and pitfalls. Semin Pediatr Surg 2014;23(05): 298-302

7 Tsukimori K, Nakanami N, Fukushima K, Yoshimura T, Hikino S, Nakano H. Pleural fluid/serum immunoglobulin ratio is a diagnostic marker for congenital chylothorax in utero. J Perinat Med 2006;34(04):313-317 
8 van Straaten HL, Gerards LJ, Krediet TG. Chylothorax in the neonatal period. Eur J Pediatr 1993;152(01):2-5

9 Villena V, de Pablo A, Martín-Escribano P. Chylothorax and chylous ascites due to heart failure. Eur Respir J 1995;8(07):1235-1236

10 Soysal DE, Hizar Turan S, Ozmen M, et al. A rare case of systemic lupus erythematosus with chylous ascites and chylothorax. Case Rep Rheumatol 2013;2013:797696

11 Lin YJ, Chen DY, Lan JL, Hsieh TY. Chylothorax as the initial presentation of systemic lupus erythematosus: a case report. Clin Rheumatol 2007;26(08):1373-1374
12 Lee CK, Han JM, Lee KN, et al. Concurrent occurrence of chylothorax, chylous ascites, and protein-losing enteropathy in systemic lupus erythematosus. J Rheumatol 2002;29(06):1330-1333

13 Dohmoto T, Kuranobu Y, Mino Y, et al. Case report of congenital chylothorax effectively treated by prednisolone administration. . [Article in Japanese]Journal of Japan Society for Premature and Newborn Medicine 2009;21:279-285

14 Schroth M, Meißner U, Cesnjevar R, et al. Plasmatic [corrected] factor XIII reduces severe pleural effusion in children after openheart surgery. Pediatr Cardiol 2006;27(01):56-60 